\title{
CORRELAÇÕES AUDIOMÉTRICAS DO QUESTIONÁRIO DE HANDICAP AUDITIVO PARA ADULTOS
}

\section{Audiometric correlations of the Hearing Handicap Inventory for Adults}

\author{
Ivanildo Inacio de Lima ${ }^{(1)}$, Camila Piccini Aiello ${ }^{(2)}$, Deborah Viviane Ferrari ${ }^{(3)}$
}

\begin{abstract}
RESUMO
Objetivo: verificar as relações entre a autopercepção do handicap auditivo (restrição de participação), os achados audiométricos e dados sócio-demográficos em adultos deficientes auditivos. Comparar os resultados da percepção do handicap entre homens e mulheres. Método: participaram deste estudo, 113 adultos jovens portadores de deficiência auditiva pós-lingual, neurossenssorial bilateral de graus variados. O questionário de Handicap Auditivo para Adultos (HHIA) foi aplicado no formato de entrevista. A pontuação total e das subescalas "social" e "emocional", do questionário foram correlacionadas com as médias dos limiares audiométricos (ISO, baixa, média e alta frequência) e limiar de reconhecimento de fala (LRF). Também foi obtida correlação entre as pontuações do questionário e tempo de surdez, escolaridade e nível sócio-econômico. A comparação dos resultados do HHIA entre homens e mulheres foi realizada pelo teste t de Student. Resultados: correlações fracas, porém significantes, foram obtidas entre os dados audiométricos e a pontuação do HHIA. Não houve correlação entre o tempo de surdez, escolaridade e nível sócio-econômico com a pontuação do questionário. Não houve diferença significativa da pontuação entre homens e mulheres. Conclusão: os dados deste estudo reforçam a necessidade de utilização de um instrumento de avaliação da restrição de participação, já que esta não pode ser inferida a partir dos dados audiológicos e/ou sócio-demográficos.
\end{abstract}

DESCRITORES: Audição; Perda Auditiva; Questionários; Audiometria

\section{INTRODUÇÃO}

Os ajustes emocionais e psicológicos frente a uma perda auditiva na vida adulta são altamente variáveis e dependentes das experiências de vida, das expectativas relacionadas à saúde, do modo costumeiro de gerenciar desafios, da habilidade de se adaptar a um dado conjunto de limitações e do grau de sociabilidade do indivíduo. Assim, pessoas com perdas auditivas semelhantes podem experimentar diferentes dificuldades comunicativas,

(1) Fonoaudiólogo; Discente do Programa de Mestrado em Fonoaudiologia da Faculdade de Odontologia de Bauru da Universidade de São Paulo, FOB-USP, Bauru, SP, Brasil.

(2) Discente do Curso de Graduação em Fonoaudiologia da Faculdade de Odontologia de Bauru da Universidade de São Paulo, FOB-USP, Bauru, SP, Brasil.

(3) Fonoaudióloga; Professora do Departamento de Fonoaudiologia da Faculdade de Odontologia de Bauru da Universidade de São Paulo, FOB-USP, Bauru, SP, Brasil; Doutora em Neurociências e Comportamento pelo Instituto de Psicologia da Universidade de São Paulo.

Conflito de interesses: inexistente sociais e emocionais em situações de vida diária e ter percepções distintas da sua qualidade de vida ${ }^{1,2}$.

Atualmente, a Organização Mundial de Saúde utiliza um modelo biossocial para redefinir os conceitos de "incapacidade" e "handicap". A limitação de atividade (incapacidade) é caracterizada como as consequências da deficiência no rendimento funcional, ou seja, na execução de uma tarefa ou ação. Já a restrição de participação (handicap) diz respeito ao envolvimento nas situações de vida e reflete a adaptação do indivíduo ao meio ambiente como resultado da perda de audição e da incapacidade. Este modelo conceitual indica que não existe uma associação direta entre a perda de audição e o grau de limitação de atividade ou restrição de participação vivenciada por um dado indivíduo ${ }^{3}$.

Pelo exposto, embora essenciais, os dados da avaliação audiológica podem ser insuficientes para selecionar candidatos a um programa de intervenção ou planejar estratégias de reabilitação ${ }^{4,5}$. 
Questionários de auto-avaliação vêm sendo desenvolvidos para quantificar a percepção do handicap, sendo utilizados na clínica para identificar as necessidades específicas de tratamento ou avaliar os resultados da intervenção ${ }^{6,7}$.

A literatura relata correlações fracas ou modestas entre o resultado de questionários de auto-avaliação do handicap e limiares auditivos e índices de reconhecimento de fala, em adultos e idosos ${ }^{8}$. Uma exceção foi um estudo realizado com trabalhadores italianos expostos a ruído, no qual forte correlação foi encontrada entre a percepção das consequências emocionais e os limiares audiométricos ${ }^{9}$.

Usualmente as comparações entre dados audiométricos e handicap é realizada, tendo como base os limiares auditivos da melhor orelha, na região de $500 \mathrm{~Hz}, 1 \mathrm{k}$ e $2 \mathrm{kHz}$. No entanto, estas correlações aumentam quando considerados os limiares auditivos nas frequências de $1 \mathrm{k}$ a $4 \mathrm{kHz}$, provavelmente em função da importância desta região de frequência na percepção das consoantes ${ }^{10}$. Um estudo demonstrou que indivíduos com perdas mais significantes nas frequências altas relataram maior percepção do handicap ${ }^{11}$.

Neste sentido, o objetivo do presente estudo foi verificar a relação entre a autopercepção do handicap, a média dos limiares audiométricos em diferentes regiões de frequência e o limiar de reconhecimento de fala em adultos com deficiência auditiva neurossensorial. As relações do handicap com dados demográficos também foram observadas.

\section{MÉTODO}

Estudo descritivo transversal de associação, desenvolvido na Clínica de Fonoaudiologia da Faculdade de Odontologia de Bauru da Universidade de São Paulo (FOB-USP). Foram avaliados 113 indivíduos (52 mulheres e 61 homens) com idades variando entre 21 e 64 anos (média de 53,6 anos) que apresentavam perda auditiva neurossensorial bilateral, pós-lingual, de graus variados e que não tinham nenhuma experiência prévia com o uso do aparelho de amplificação sonora individual (AASI). Todos os indivíduos estavam regularmente matriculados na Clínica de Fonoaudiologia da FOB-USP e participaram como voluntários, tendo assinado o termo de consentimento livre e esclarecido.

A média e desvio padrão dos limiares audiométricos da orelha com melhores limiares auditivos dos participantes encontra-se na Figura 1.

A Tabela 1 mostra os dados demográficos dos participantes. A classificação sócio-econômica foi obtida a partir do protocolo Instrumental de Classificação Sócio-Econômica contido nos prontuários dos indivíduos ${ }^{12}$. Para a classificação do grau da perda auditiva foi utilizada a média dos limiares audiométricos nas frequências de $500,1 \mathrm{k}, 2 \mathrm{k}$ e $4 \mathrm{kHz}$ (média ISO), sendo considerada a orelha com melhores limiares audiométricos. Cabe ressaltar que em virtude do elevado grau de perda auditiva seis indivíduos não conseguiram realizar a avaliação do limiar de reconhecimento de fala. A duração da deficiência auditiva foi considerada conforme relato do

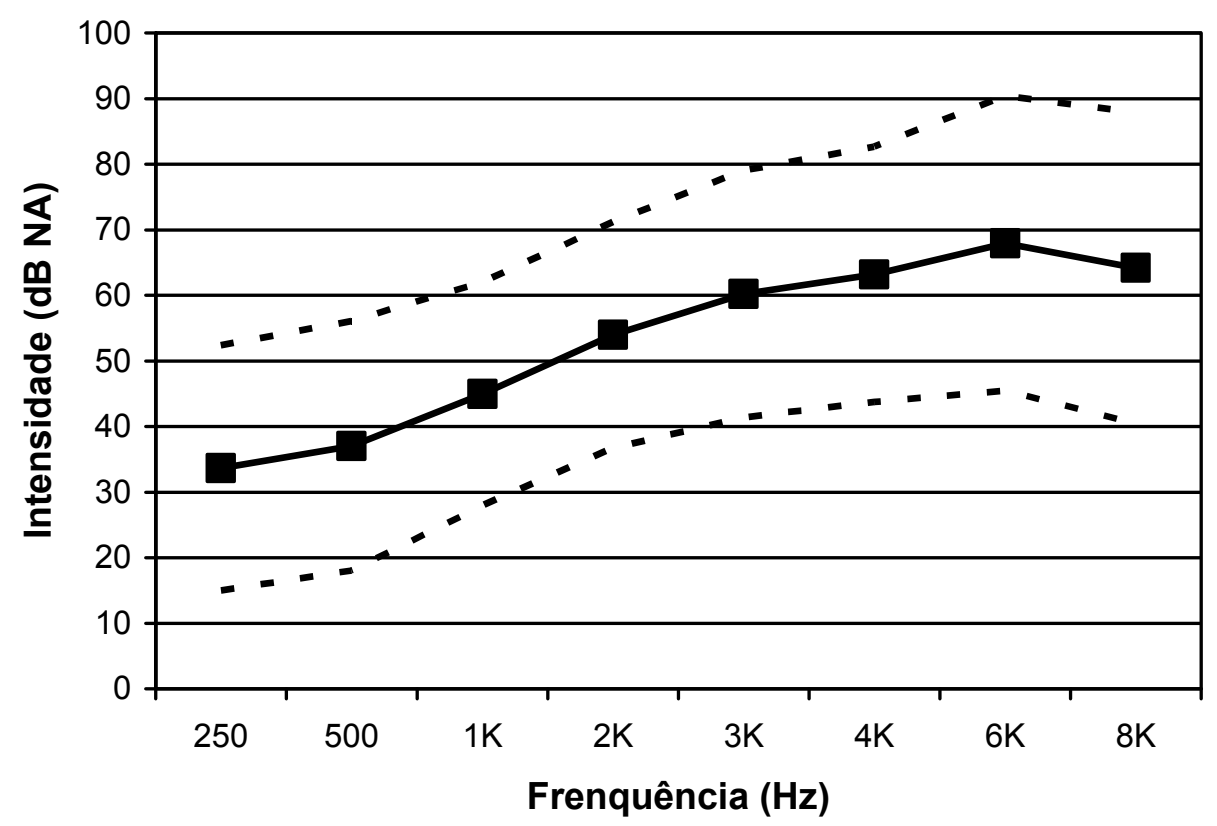

Figura 1 - Média e desvio padrão dos limiares audiométricos dos participantes ( $n=113)$ 
Tabela 1 - Dados demográficos dos participantes

\begin{tabular}{|c|c|c|c|c|c|c|c|c|c|c|c|c|c|c|c|c|c|c|c|c|c|c|c|}
\hline & \multicolumn{4}{|c|}{$\begin{array}{c}\text { Média Limiar dB } \\
\text { NA }(500,1 k, 2 k \text {, } \\
4 \mathrm{kHz})\end{array}$} & \multicolumn{4}{|c|}{$\begin{array}{c}\text { Limiar de } \\
\text { Reconhecimento } \\
\text { de Fala - LRF (dB } \\
\text { NA) }\end{array}$} & \multicolumn{5}{|c|}{$\begin{array}{c}\text { Duração da } \\
\text { deficiência auditiva } \\
\text { (meses) }\end{array}$} & \multicolumn{6}{|c|}{ Escolaridade } & \multicolumn{4}{|c|}{$\begin{array}{l}\text { Classificação } \\
\text { Sócio- } \\
\text { Econômica }\end{array}$} \\
\hline & 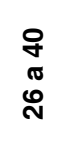 & 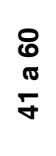 & $\begin{array}{l}\infty \\
\infty \\
\pi \\
\sigma\end{array}$ & $\begin{array}{c}\mathbf{\infty} \\
\wedge\end{array}$ & $\begin{array}{l}0 \\
\text { m } \\
\pi \\
12\end{array}$ & 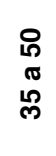 & $\begin{array}{l}0 \\
\pi \\
\kappa \\
\kappa\end{array}$ & $\begin{array}{l}8 \\
\text { o } \\
\text { n }\end{array}$ & $\begin{array}{l}\dot{E} \\
\stackrel{\mathbf{N}}{\Phi} \\
\frac{\pi}{\pi}\end{array}$ & 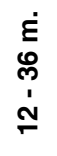 & $\begin{array}{l}\dot{\varepsilon} \\
0 \\
1 \\
\hat{m}\end{array}$ & $\begin{array}{l}\dot{E} \\
\stackrel{\text { Tे}}{1} \\
\frac{1}{0}\end{array}$ & $\begin{array}{l}\dot{E} \\
\stackrel{\text { }}{\Lambda} \\
\Lambda\end{array}$ & $\begin{array}{l}\frac{4}{4} \\
0 \\
\frac{10}{2}\end{array}$ & $\begin{array}{l}\dot{\dot{j}} \\
\dot{\mathrm{c}} \\
\dot{3}\end{array}$ & $\begin{array}{l}\dot{0} \\
\dot{0} \\
\grave{5} \\
\dot{L}\end{array}$ & $\begin{array}{l}\dot{0} \\
\frac{0}{8} \\
\dot{e}\end{array}$ & $\begin{array}{l}\dot{0} \\
\stackrel{0}{0} \\
\stackrel{0}{0} \\
\Sigma\end{array}$ & 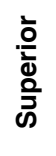 & 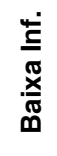 & $\begin{array}{l}\dot{\infty} \\
\mathbb{\Xi} \\
\text { 產 } \\
\tilde{\infty}\end{array}$ & 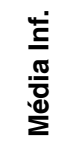 & $\stackrel{\frac{\pi}{8}}{\frac{\pi}{\Sigma}}$ \\
\hline $\begin{array}{c}\text { № de } \\
\text { Indivíduos }\end{array}$ & 32 & 62 & 13 & 6 & 14 & 62 & 24 & 7 & 2 & 11 & 11 & 26 & 56 & 3 & 47 & 10 & 10 & 30 & 8 & 13 & 76 & 19 & 4 \\
\hline Total & \multicolumn{4}{|c|}{113} & \multicolumn{4}{|c|}{107} & \multicolumn{5}{|c|}{106} & \multicolumn{6}{|c|}{108} & \multicolumn{4}{|c|}{112} \\
\hline
\end{tabular}

indivíduo no momento da anamnese. A informação quanto à duração da deficiência auditiva e escolaridade não constavam no prontuário de sete e cinco participantes, respectivamente.

O Questionário de Handicap Auditivo para Adultos (Hearing Handicap Inventory for Adults $\mathrm{HHIA}$ ) traduzido para o português brasileiro (Figura 2) foi aplicado 8,13 . Este questionário é composto por 25 questões que quantificam os efeitos sociais (12 questões) e emocionais (13 questões) da deficiência auditiva em indivíduos com idade inferior a 65 anos.

A aplicação do questionário HHIA foi realizada no formato de entrevista, após a realização do diagnóstico audiológico, quando o participante iniciava os procedimentos de seleção do AASI. O avaliador leu as 25 questões juntamente com o participante que foi solicitado a assinalar a resposta que julgasse mais adequada. Três alternativas estavam disponíveis para cada questão: "sim" (equivalente a 4 pontos), "às vezes" (equivalente a 2 pontos) e "não" (equivalente a 0 ponto).

A pontuação do questionário HHIA foi realizada manualmente, sendo calculada a pontuação total (somatória dos pontos para as 25 questões) bem como das sub-escalas emocional e social, separadamente. A pontuação total pode variar de $0 \mathrm{a}$ 100. Valores mais próximos de 100 indicam maior percepção do handicap auditivo. De acordo com a pontuação o questionário indica se o indivíduo não apresenta percepção do handicap (entre 0 e 16\%) ou se apresenta percepção leve/moderada (18 a $42 \%$ ) ou severa (acima de $42 \%$ ) do handicap.

O trabalho foi analisado e aprovado pelo Comitê de Ética em Pesquisa da Faculdade de Odontologia de Bauru, Universidade de São Paulo - FOB/USP (processo $n^{\circ}$ 041/2007).

A análise estatística foi realizada por meio do software Stata. Foi calculado o coeficiente de correlação de Pearson entre a pontuação total e das subescalas emocional e social do HHIA com a média ISO, médias das frequências baixas $(0,25,0,5 \mathrm{e}$ $1 \mathrm{kHz}$ ), médias $(0,5,1$ e $2 \mathrm{kHz}$ ) e altas (1k, 2k e 4 $\mathrm{kHz}$ ) dos limiares da orelha com melhores limiares auditivos. O coeficiente de correlação de Spearman foi utilizado para verificar as relações entre a escolaridade e nível sócio-econômico com as pontuações do questionário HHIA. A comparação da pontuação do questionário entre homens e mulheres foi realizada por meio do teste t de Student. Para todos os casos foi adotado nível de significância de 5\%.

\section{RESULTADOS}

A média e desvio padrão da pontuação total e das subescalas do HHIA encontram-se na Figura 3.

A Tabela 2 mostra a correlação entre a pontuação do questionário HHIA e as médias dos limiares audiométricos e do reconhecimento de fala (LRF).

A Tabela 3 mostra as correlações entre a pontuação do questionário HHIA com os dados demográficos dos participantes.

As comparações da pontuação do questionário entre homens e mulheres por meio do teste $t$ de Student encontram-se na Figura 4.

\section{DISCUSSÃO}

Foram observadas correlações positivas fracas, porém significantes, entre as médias dos limiares audiométricos e as pontuações das subescalas emocional e social e total do HHIA (Tabela 2). A literatura também relata correlações fracas e significantes entre a média dos limiares audiométricos e o handicap auditivo, sendo estas ao redor de 0,38 e $0,34^{8-10}$.

No presente estudo também foi encontrada uma correlação fraca e significativa entre o limiar de reconhecimento de fala e as pontuações: total 
Nome:

Data:

Instruções: O questionário a seguir contém 25 perguntas. Você deverá escolher apenas uma resposta para cada pergunta, colocando um $(\mathrm{X})$ naquela que julgar adequada. Algumas perguntas são parecidas, mas na realidade têm pequenas diferenças que permitem uma melhor avaliação das respostas. Não há resposta certa ou errada. Você deverá marcar aquela que julgar ser a mais adequada ao seu caso ou situação.

\begin{tabular}{|c|c|c|c|c|}
\hline & & $\underset{(4)}{\operatorname{Sim}}$ & $\begin{array}{l}\text { Às vezes } \\
\text { (2) }\end{array}$ & $\begin{array}{c}\text { Não } \\
(0)\end{array}$ \\
\hline S-1 & $\begin{array}{l}\text { A dificuldade em ouvir faz você usar o telefone menos vezes do que } \\
\text { gostaria? }\end{array}$ & & & \\
\hline $\mathrm{E}-2$ & $\begin{array}{l}\text { A dificuldade em ouvir faz você se sentir constrangido ou sem jeito } \\
\text { quando é apresentado a pessoas desconhecidas? }\end{array}$ & & & \\
\hline S-3 & A dificuldade em ouvir faz você evitar grupos de pessoas? & & & \\
\hline E-4 & A dificuldade em ouvir faz você ficar irritado? & & & \\
\hline E-5 & $\begin{array}{l}\text { A dificuldade em ouvir faz você se sentir frustrado ou insatisfeito } \\
\text { quando conversa com pessoas da sua família? }\end{array}$ & & & \\
\hline S-6 & $\begin{array}{l}\text { A diminuição da audição causa outras dificuldades quando você vai a } \\
\text { uma festa ou reunião social? }\end{array}$ & & & \\
\hline E-7 & $\begin{array}{l}\text { A dificuldade em ouvir faz você se sentir frustrado ao conversar com } \\
\text { os colegas de trabalho? }\end{array}$ & & & \\
\hline S-8 & Você sente dificuldade em ouvir quando vai ao cinema ou teatro? & & & \\
\hline E-9 & $\begin{array}{l}\text { Você se sente prejudicado ou diminuído devido a sua dificuldade em } \\
\text { ouvir? }\end{array}$ & & & \\
\hline S-10 & $\begin{array}{l}\text { A diminuição da audição causa dificuldades quando visita amigos, } \\
\text { parentes ou vizinhos? }\end{array}$ & & & \\
\hline S-11 & $\begin{array}{l}\text { A dificuldade em ouvir faz com que você tenha problemas para ouvir/ } \\
\text { entender os colegas de trabalho? }\end{array}$ & & & \\
\hline $\mathrm{E}-12$ & A dificuldade em ouvir faz você ficar nervoso? & & & \\
\hline S-13 & $\begin{array}{l}\text { A dificuldade em ouvir faz você visitar amigos, parentes ou vizinhos } \\
\text { menos do que gostaria? }\end{array}$ & & & \\
\hline E-14 & $\begin{array}{l}\text { A dificuldade em ouvir faz você ter discussões ou brigas com a sua } \\
\text { família? }\end{array}$ & & & \\
\hline S-15 & $\begin{array}{l}\text { A diminuição da audição causa dificuldades para assistir TV ou ouvir } \\
\text { rádio? }\end{array}$ & & & \\
\hline S-16 & $\begin{array}{l}\text { A dificuldade em ouvir faz com que você saia para fazer compras } \\
\text { menos vezes do que gostaria? }\end{array}$ & & & \\
\hline E-17 & $\begin{array}{l}\text { A dificuldade em ouvir deixa você de alguma maneira chateado ou } \\
\text { aborrecido? }\end{array}$ & & & \\
\hline$E-18$ & A dificuldade em ouvir faz você preferir ficar sozinho? & & & \\
\hline S-19 & $\begin{array}{l}\text { A dificuldade em ouvir faz você querer conversar menos com as } \\
\text { pessoas de sua família? }\end{array}$ & & & \\
\hline $\mathrm{E}-20$ & $\begin{array}{l}\text { Você acha que a dificuldade em ouvir diminui ou limita de alguma } \\
\text { forma sua vida pessoal ou social? }\end{array}$ & & & \\
\hline S-21 & $\begin{array}{l}\text { A diminuição da audição Ihe causa dificuldades quando você está em } \\
\text { um restaurante com familiares ou amigos? }\end{array}$ & & & \\
\hline $\mathrm{E}-22$ & A dificuldade em ouvir faz você se sentir triste ou deprimido? & & & \\
\hline S-23 & $\begin{array}{l}\text { A dificuldade em ouvir faz você assistir TV ou ouvir rádio menos que } \\
\text { gostaria? }\end{array}$ & & & \\
\hline E-24 & $\begin{array}{l}\text { A dificuldade em ouvir faz você se sentir constrangido ou menos à } \\
\text { vontade quando conversa com amigos? }\end{array}$ & & & \\
\hline E-25 & $\begin{array}{l}\text { A dificuldade em ouvir faz você se sentir isolado ou deixado de lado } \\
\text { num grupo de pessoas? }\end{array}$ & & & \\
\hline & PARA USO DO CLÍNICO: Pontuação Total: & E: & S: & \\
\hline
\end{tabular}

Figura 2 - The Hearing Handicap Inventory for Adults 


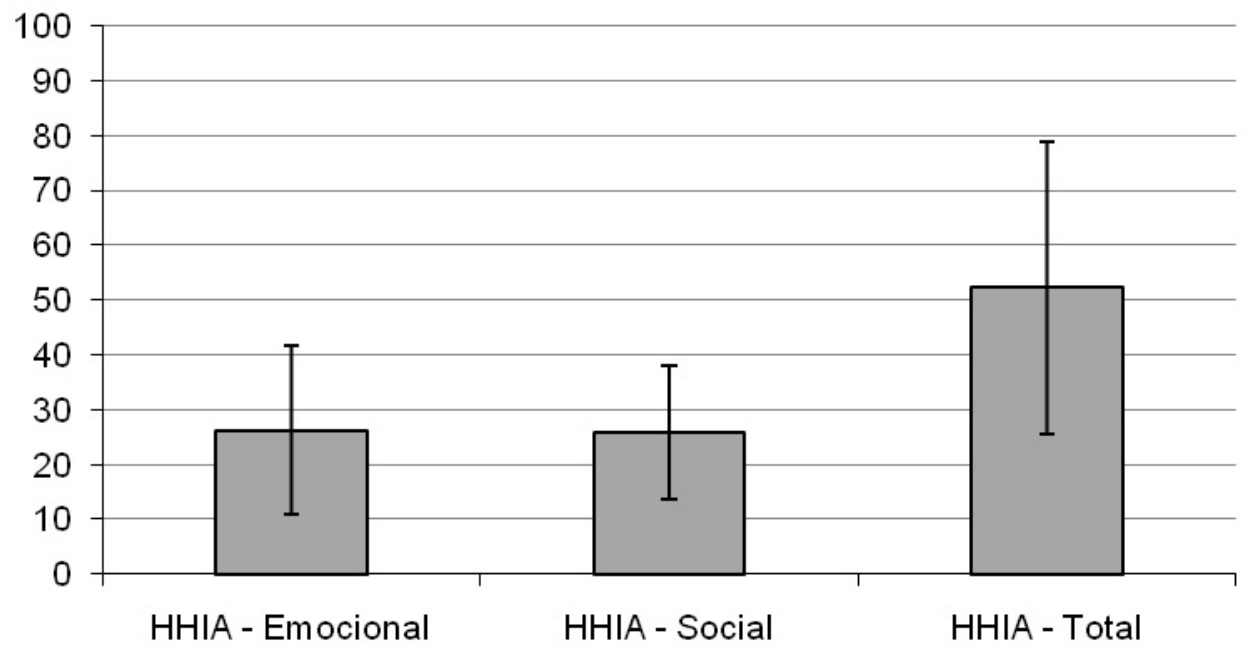

Figura 3 - Média e desvio padrão das pontuações das sub-escalas emocional e social e total do questionário HHIA $(n=113)$

Tabela 2 - Correlação de Pearson e significância entre a pontuação do questionário e a média dos limiares audiométricos $(n=113)$ e do limiar de reconhecimento de fala $(n=107)$

\begin{tabular}{|c|c|c|c|}
\hline $\begin{array}{l}\text { Média dos limiares } \\
\text { auditivos (dB NA) }\end{array}$ & HHIA - Emocional & HHIA - Social & HHIA - Total \\
\hline \multirow{2}{*}{ ISO } & $r=0,26$ & $r=0,27$ & $r=0,28$ \\
\hline & $p=0,01^{*}$ & $p=0,00^{*}$ & $p=0,00^{*}$ \\
\hline \multirow{2}{*}{ Baixas } & $r=0,27$ & $r=0,31$ & $r=0,30$ \\
\hline & $P=0,00^{*}$ & $p=0,00^{*}$ & $p=0,00^{*}$ \\
\hline \multirow{2}{*}{ Médias } & $r=0,23$ & $r=0,26$ & $r=0,25$ \\
\hline & $P=0,01^{*}$ & $p=0,01^{*}$ & $p=0,01^{*}$ \\
\hline \multirow{2}{*}{ Altas } & $r=0,23$ & $r=0,22$ & $r=0,24$ \\
\hline & $p=0,01^{*}$ & $p=0,02^{*}$ & $p=0,01^{*}$ \\
\hline \multicolumn{4}{|l|}{$\begin{array}{l}\text { Reconhecimento de } \\
\text { fala (dB NA) }\end{array}$} \\
\hline LRF & $\begin{aligned} r & =0,26 \\
P & =0,01^{*}\end{aligned}$ & $\begin{array}{l}r=0,22 \\
p=0,22\end{array}$ & $\begin{aligned} r & =0,25 \\
p & =0,01^{*}\end{aligned}$ \\
\hline
\end{tabular}

Tabela 3 - Correlações entre a pontuação do questionário e os dados demográficos dos participantes $(n=113)$

\begin{tabular}{|c|c|c|c|c|c|c|c|c|c|c|c|c|c|c|c|c|c|c|c|c|c|c|c|}
\hline & \multicolumn{4}{|c|}{$\begin{array}{c}\text { Média Limiar dB } \\
\text { NA }(500,1 \mathrm{k}, 2 \mathrm{k}, \\
4 \mathrm{kHz})\end{array}$} & \multicolumn{4}{|c|}{$\begin{array}{c}\text { Limiar de } \\
\text { Reconhecimento } \\
\text { de Fala - LRF (dB } \\
\text { NA) }\end{array}$} & \multicolumn{5}{|c|}{$\begin{array}{l}\text { Duração da } \\
\text { deficiência auditiva } \\
\text { (meses) }\end{array}$} & \multicolumn{6}{|c|}{ Escolaridade } & \multicolumn{4}{|c|}{$\begin{array}{l}\text { Classificação } \\
\text { Sócio- } \\
\text { Econômica }\end{array}$} \\
\hline & $\begin{array}{l}\text { q } \\
\pi \\
\mathscr{N}\end{array}$ & $\begin{array}{l}8 \\
\frac{8}{\pi}\end{array}$ & $\begin{array}{l}\stackrel{8}{\infty} \\
\pi \\
\bar{\sigma}\end{array}$ & $\begin{array}{l}0 \\
1 \\
\Lambda\end{array}$ & $\begin{array}{l}\text { D } \\
\text { జ } \\
\text { [2 }\end{array}$ & $\begin{array}{l}\text { in } \\
\text { o } \\
\text { ம }\end{array}$ & $\begin{array}{l}R \\
\pi \\
i \\
i n\end{array}$ & $\begin{array}{l}8 \\
8 \\
\pi \\
\text { R }\end{array}$ & 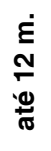 & $\begin{array}{l}\dot{E} \\
0 \\
m \\
\dot{N}\end{array}$ & $\begin{array}{l}\dot{E} \\
8 \\
1 \\
\hat{m}\end{array}$ & $\begin{array}{l}\dot{E} \\
\stackrel{2}{N} \\
1 \\
\frac{1}{0}\end{array}$ & $\begin{array}{l}\dot{E} \\
\stackrel{N}{N} \\
\wedge\end{array}$ & $\begin{array}{l}\frac{4}{4} \\
\frac{2}{20} \\
\frac{10}{2}\end{array}$ & $\begin{array}{l}\dot{i} \\
\dot{\grave{c}} \\
\stackrel{5}{\mathbf{L}}\end{array}$ & $\begin{array}{l}\dot{j} \\
\dot{0} \\
\stackrel{\partial}{5}\end{array}$ & 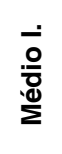 & $\begin{array}{l}\dot{0} \\
\stackrel{0}{0} \\
\stackrel{\circ}{\varrho}\end{array}$ & $\begin{array}{l}\text { 흔 } \\
\text { ஸํํำ }\end{array}$ & 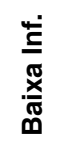 & 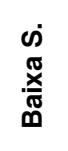 & 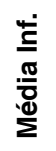 & $\frac{\pi}{\frac{\pi}{\delta}}$ \\
\hline $\begin{array}{c}\text { № de } \\
\text { Indivíduos }\end{array}$ & 32 & 62 & 13 & 6 & 14 & 62 & 24 & 7 & 2 & 11 & 11 & 26 & 56 & 3 & 47 & 10 & 10 & 30 & 8 & 13 & 76 & 19 & 4 \\
\hline Total & \multicolumn{4}{|c|}{113} & \multicolumn{4}{|c|}{107} & \multicolumn{5}{|c|}{106} & \multicolumn{6}{|c|}{108} & \multicolumn{4}{|c|}{112} \\
\hline
\end{tabular}


$\square$ Homens $\square$ Mulheres

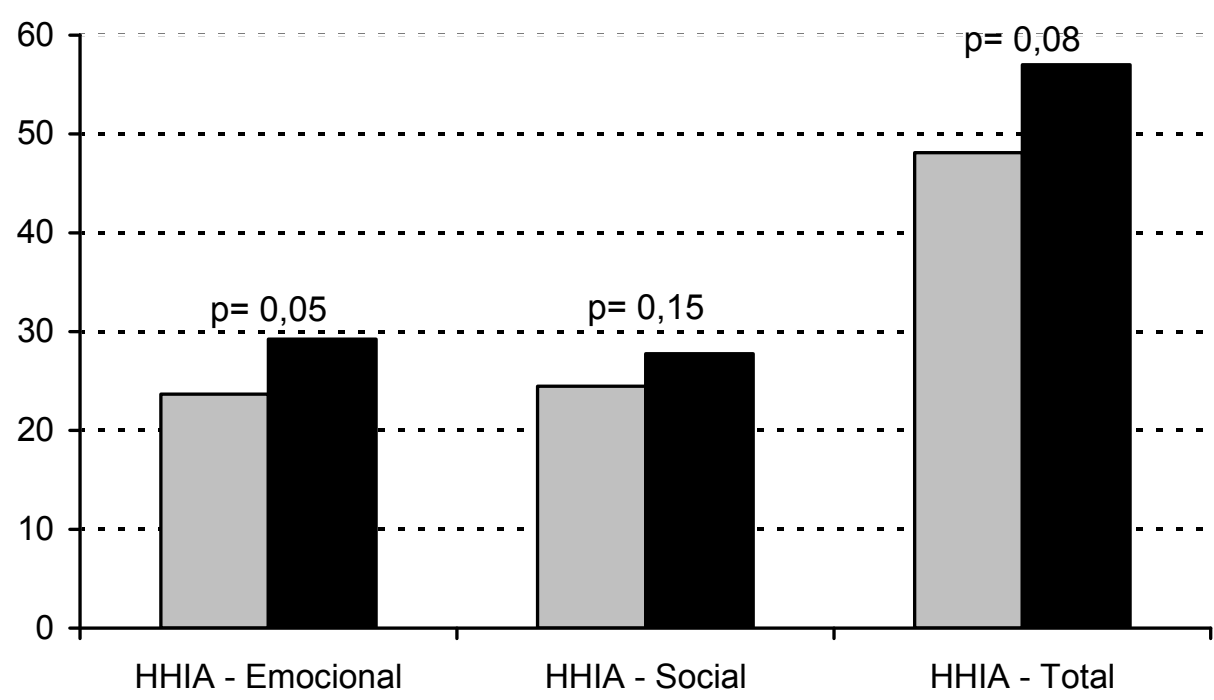

Figura 4 - Média das pontuações das subescalas emocional e social e total do questionário HHIA e a significância entre os sexos $(p<0,05)$

e da sub-escala emocional do HHIA, sendo esta semelhante à encontrada na literatura ${ }^{8}$.

Os achados deste estudo devem ser interpretados com cautela. Embora estatisticamente significantes as correlações encontradas no presente estudo foram muito fracas para se afirmar que os dados audiométricos podem predizer o handicap para um determinado indivíduo. De fato, em um estudo com 343 participantes a análise de regressão mostrou que apenas $28,4 \%$ da variância dos resultados de avaliação do handicap podem ser preditos por fatores como a idade, a média dos limiares audiométricos, a auto-percepção da severidade dos problemas auditivos ${ }^{3}$.

A relação imperfeita entre os limiares auditivos e o handicap dá-se pelo fato de que este último envolve uma complexa e multifatorial relação do indivíduo com o seu meio. Isto indica que as medidas objetivas e outras variáveis tradicionalmente acessíveis são insuficientes para explicar todos os aspectos de como os indivíduos experimentam a vida diária com a deficiência auditiva ${ }^{3,5}$.

Estes dados trazem reflexões importantes para o modelo atualmente utilizado para seleção de candidatos à intervenção. No Brasil, o médico otorrinolaringologista é quem define o tipo de tratamento para um dado paciente. Assim, quando necessário, aconselhará $o$ deficiente auditivo sobre a possibilidade de tratamento clínico/reabilitativo, como por exemplo, o uso do AASI. A partir daí é feito o encaminhamento do indivíduo para um fonoaudiólogo, que realizará os procedimentos de seleção e adaptação deste dispositivo. Ressalta-se que tradicionalmente o critério médico utilizado para recomendar ou não a intervenção são os exames audiométricos como a audiometria tonal e os resultados de reconhecimento da fala. No entanto, o valor preditivo de tais testes para estimar o handicap é muito pequeno. Estudos baseados em evidência também demonstram que estes dados isolados não predizem também se o indivíduo obterá ou não benefício com o uso da amplificação ${ }^{14,15}$.

Mesmo na clínica fonoaudiológica, os dados audiométricos são frequentemente utilizados em detrimento dos questionários de auto-avaliação. Uma pesquisa realizada na cidade de São Paulo mostrou que $75 \%$ dos fonoaudiólogos não utilizam questionários de auto-avaliação. Dentre os motivos para tal, foram apontados o grande tempo despendido na aplicação destes instrumentos, falta de conhecimento ou, ainda, a não inclusão de questionários no protocolo de seleção adotado pelo local de trabalho do profissional ${ }^{16}$.

Foram também observadas correlações muito fracas e não significantes entre as variáveis: idade, duração da perda auditiva, escolaridade e nível sócio econômico e as pontuações do HHIA. Um estudo demonstrou que a idade e o status ocupacional do indivíduo influenciam a percepção do handicap ${ }^{11}$. Os resultados do presente estudo podem ser explicados pela característica mais homogênea da população, tendo em vista que $67 \%$ dos participantes possuíam classificação sócio- 
econômica baixo-superior e 55\% eram analfabetos ou possuíam ensino fundamental.

Embora não tenham feito parte dos objetivos deste estudo é interessante observar os dados da duração da deficiência auditiva. Todos os participantes deste estudo não tinham nenhum tipo de experiência com o uso do AASI e procuraram atendimento pela primeira vez. Ressalta-se que apenas dois de 106 participantes relataram o aparecimento das dificuldades auditivas há um ano ou menos. Ou seja, apenas $2,6 \%$ dos participantes procuraram atendimento no primeiro ano de aparecimento da deficiência auditiva. Cerca de $50 \%$ dos participantes apresentavam a perda auditiva há dez anos ou mais antes de procurar o tratamento. São vários os fatores que levam o indivíduo a postergar a procura pelo tratamento; dentre eles a incapacidade do indivíduo de reconhecer os sinais de uma perda auditiva ou mesmo processos psicológicos e mecanismos de enfrentamento como a negação ${ }^{17}$. A dificuldade para acesso ao serviço de saúde auditiva também pode ser determinante para o tempo entre o aparecimento da perda auditiva e a procura pelo tratamento.
Os dados da Figura 4 mostram que, em média, as mulheres apresentaram maior handicap que os homens, no entanto esta diferença não foi estatisticamente significante. Resultados semelhantes também foram observados em outros estudos nacionais e internacionais ${ }^{3,11,18}$.

\section{CONCLUSÃO}

Os resultados deste estudo reforçam o fato de que os dados audiométricos e demográficos não permitem estimar o handicap de modo que tenha utilidade clínica para indicação ou planejamento de algum tipo de intervenção para um dado paciente. Sabendo-se que este processo frequentemente baseia-se apenas nos resultados dos limiares auditivos, tais achados também servem de alerta para a revisão das práticas clínicas, com a inclusão de algum instrumento específico para a avaliação do handicap. A combinação destas informações será de auxílio para o profissional da área audiológica e para o paciente fazerem escolhas apropriadas relativas à necessidade de tratamento.

\begin{abstract}
Purpose: to check the relations between self-perceived hearing handicap (participation restriction), audiometric findings and socio-demographic data in hearing impaired adults and compare the results between men and women. Method: 113 adults with post lingual sensorineural bilateral hearing loss of varying degrees took part in the study. The Hearing Handicap Inventory for Adults (HHIA) was applied in the interview format. We obtained the correlation coefficient between total HHIA as well as the "social" one and "emotional" subscales scores with the mean audiometric thresholds (ISO, low, medium and high frequencies) and speech recognition thresholds (SRT). The HHIA scores were also correlated with the duration of deafness, educational level and socio-economic status. The HHIA scores between men and women were compared by means of the Student $t$ test. Results: weak but significant correlations were obtained between audiometric data and HHIA scores. There was no correlation between duration of deafness, educational level and socioeconomic status with HHIA scores. There was no significant difference in scores between men and women. Conclusion: data from this study reinforce the need for using an instrument for evaluating the participation restriction, since it cannot be inferred from the audiometric data and/or socio-demographic factors.
\end{abstract}

KEYWORDS: Hearing; Hearing Loss; Questionnaires; Audiometry

\section{REFERÊNCIAS}

1. Hallberg LR, Hallberg U, Kramer SE. Self-reported hearing difficulties, communication strategies and psychological general well-being (quality of life) in patients with acquired hearing impairment. Disabil Rehabil. 2008;30(3):203-12.
2. Helvik AS, Jacobsen G, Hallberg LR. Psychological well-being of adults with acquired hearing impairment. Disabil Rehabil. 2006 May 15;28(9):535-45.

3. Helvik AS, Jacobsen G, Wennberg S, Arnesen $H$, Ringdahl A, Hallberg LR. Activity limitation and participation restriction in adults seeking hearing aid 
fitting and rehabilitation. Disabil Rehabil. 2006 Mar; 28(5):281-8.

4. AAA: American Academy of Audiology. Guidelines for the audiologic management of adult hearing impairment. Audiology Today. 2006 Sep-Oct; 18(5):32-6.

5. Palmer CV, Solodar HS, Hurley WR, Byrne DC, Williams KO. Self-perception of hearing ability as a strong predictor of hearing aid purchase. J Am Acad Audiol. 2009 Jun;20(6):341-7.

6. Saccone PA, Steiger JR. Hearing handicap among adult residents of an urban homeless shelter. J Health Care Poor Underserved. 2007 Feb; 18(1):161-72.

7. Abrams HB, McArdle R, Chisolm TH. From outcomes to evidence: establishing best practices for audiologists. Semin Hear 2005; 26(3):157-69.

8. Newman CW, Weinstein BE, Jacobson GP, Hug GA. The Hearing Handicap Inventory for Adults: psychometric adequacy and audiometric correlates. Ear Hear. 1990 Dec; 11(6):430-3.

9. Monzani D, Genovese E, Palma S, Rovatti V, Borgonzoni M, Martini A. Measuring the psychosocial consequences of hearing loss in a working adult population: focus on validity and reliability of the Italian translation of the hearing handicap inventory. Acta Otorhinolaryngol Ital. 2007 Aug; 27(4):186-91.

10. Kielinen LL, Nerbonne MA. Further investigation of the relationship between hearing handicap and audiometric measures of hearing impairment. J Acad Rehabil Audiol 1990; 23:89-94.
11. Stewart M, Pankiw R, Lehman ME, Simpson $\mathrm{TH}$. Hearing loss and hearing handicap in users of recreational firearms. J. Am. Acad. Audiol. 2002 Mar; 13(3):160-8.

12. Graciano MIG, Lehfeld NAS, Neves Filho A. Instrumental de classificação sócio-econômica 1997. Serv Social Realid. 1999; 5(1):109-28.

13. Almeida K. Avaliação objetiva e subjetiva do benefício de próteses auditivas em adultos [tese]. São Paulo (SP): Universidade Federal de São Paulo - Escola Paulista de Medicina; 1998.

14. Killion MC, Gudmundsen GI. Fitting hearing aids using clinical prefitting speech measures: an evidence-based review. J Am Acad Audiol. 2005; 16(7):439-47.

15. Taylor B. Predicting real world hearing aid benefit with speech audiometry: an evidence-based review. Audiology Online [serial on the Internet] 2007 May [cited $2008 \mathrm{Jul}$ 8]; 1802: [about 10 p.]. Available from: http://www.audiologyonline.com/ articles/article_detail.asp?article_id=1802

16. Macedo LS, Pupo AC, Balieiro CR. Aplicabilidade dos questionários de auto-avaliação em adultos e idosos com deficiência auditiva. Dist Comun. 2006 Apr; 18(1):19-25.

17. Rawool VW, Keihl JM. Perception of hearing status, communication, and hearing aids among socially active older individuals. J Otolaryngol Head Neck Surg. 2008 Feb;37(1):27-42.

18. Correa GF, Russo ICP. Autopercepção do handicap em deficientes auditivos adultos e idosos. Rev. CEFAC. 1999; 1(1):54-63.

doi: 10.1590/S1516-18462010005000057

RECEBIDO EM: 17/12/2009

ACEITO EM: 12/02/2010

Endereço para correspondência

Deborah Viviane Ferrari

Faculdade de Odontologia de Bauru - USP

Departamento de Fonoaudiologia

Al. Dr. Octávio Pinheiro Brisolla 9-75

Bauru - SP

CEP: 17102-101

E-mail: deborahferrari@usp.br 